\title{
Amorphous Placement and Informed Diffusion for Timely Field Monitoring by Autonomous, Resource-Constrained, Mobile Sensors
}

\author{
HANY MORCOS \\ AZER BESTAVROS \\ IBRAHIM MATTA \\ hmorcos@cs.bu.edu \\ best@Cs.bu.edu \\ matta@cs.bu.edu \\ Computer Science Department \\ Boston University
}

\begin{abstract}
Personal communication devices are increasingly equipped with sensors for passive monitoring of encounters and surroundings. We envision the emergence of services that enable a community of mobile users carrying such resource-limited devices to query such information at remote locations in the field in which they collectively roam. One approach to implement such a service is directed placement and retrieval (DPR), whereby readings/queries about a specific location are routed to a node responsible for that location. In a mobile, potentially sparse setting, where end-to-end paths are unavailable, DPR is not an attractive solution as it would require the use of delay-tolerant (flooding-based store-carry-forward) routing of both readings and queries, which is inappropriate for applications with data freshness constraints, and which is incompatible with stringent device power/memory constraints. Alternatively, we propose the use of amorphous placement and retrieval (APR), in which routing and field monitoring are integrated through the use of a cache management scheme coupled with an informed exchange of cached samples to diffuse sensory data throughout the network, in such a way that a query answer is likely to be found close to the query origin. We argue that knowledge of the distribution of query targets could be used effectively by an informed cache management policy to maximize the utility of collective storage of all devices. Using a simple analytical model, we show that the use of informed cache management is particularly important when the mobility model results in a non-uniform distribution of users over the field. We present results from extensive simulations which show that in sparsely-connected networks, APR is more cost-effective than DPR, that it provides extra resilience to node failure and packet losses, and that its use of informed cache management yields superior performance.
\end{abstract}

\section{INTRODUCTION}

Motivation: Advances in the manufacturing and miniaturization of sensors of various modalities are making it possible for such sensors to be embedded in mobile devices such as cellular phones, handheld computers, and automotive navigational systems. Sensors are even expected to be embedded in future wearable computers to monitor vital signs [1], [19]. The communication capabilities of these devices open up the possibility of using a set of (possibly large number of) mobile devices in a given field as constituting a distributed repository of spatio-temporal sensory data. Thus, in this paper we consider parasitic applications that enable a community of mobile users carrying such devices to form ad-hoc overlay networks to query remote locations in the field in which they

$\S$ This work has been partially supported by a number of National Science Foundation grants, including CISE/CSR Award \#0720604, ENG/EFRI Award \#0735974, CISE/CNS Award \#0524477, CNS/NeTS Award \#0520166, CNS/ITR Award \#0205294, and CISE/EIA RI Award \#0202067. collectively roam - e.g., allowing a spectator in a baseball game to query the number of cell-phones (which is an estimate of the number of people) at a concession stand, or allowing a traveller to query the availability and strength of public wireless networks at various airport locations. We describe our target applications as parasitic to delineate them from the primary applications of the mobile communication device. While it is conceivable to assume that such devices may have plenty of memory and (renewable) power in support of their primary functions, it is not acceptable to assume that such resources could be tapped to support the parasitic field monitoring applications we envision. Rather, it is only prudent to assume that the resources available to such applications are quite constrained $-e . g$., the application is limited to using a small memory attached to the sensor. In this paper, and through efficient management of this limited memory, we show that the mobility of a set of sparsely deployed nodes could be leveraged to improve the recall rates for locally issued queries, posed over the field in which the nodes are roaming.

Directed versus Amorphous Placement: An important question here is related to the placement and storage of spatiotemporal samples - specifically, should each sensor node be assigned a spatiotemporal subspace for which it is responsible, or should the responsibility of the entire spatio-temporal space be shared across all nodes? We use the term "directed placement and retrieval" (DPR) to refer to the former of these approaches and the term "amorphous placement and retrieval" (APR) to refer to the latter.

DPR-like approaches have been proposed and evaluated in a number of studies in P2P networks [29], [24] as well as in sensor network (SN) settings [2], [22], [25], where they are termed as Data Centric Storage (DCS) approaches [27]. DPR simplifies query processing significantly, since a well-defined "home" for a spatio-temporal subspace makes it straightforward to route future queries over that space. In our context, using DPR, once a sample is obtained by a mobile node, storage of this sample requires its transport to the node (or locale of nodes) responsible for the spatio-temporal subspace to which this sample belongs. This could be done using any number of multi-hop ad-hoc or delay-tolerant network (DTN) routing techniques [12], [13], [21]. In a mobile, potentially sparse setting, where end-to-end paths are unavailable, DPR is not an attractive solution as it requires the use of floodingbased store-carry-forward routing of both readings and queries, 
which is inappropriate for "delay intolerant" field monitoring due to freshness requirements imposed on query results, and which is incompatible with the stringent constraints imposed on the use of device power and memory. ${ }^{1}$

Alternatively, in this paper, we propose the use of APR, whereby a reading is not associated with a locale where it must be stored, but rather such a reading could be stored in any one of the (and even replicated across multiple) mobile caches in the system. To improve the local view of nodes, upon meeting a new neighbor, nodes exchange a small number of samples. This exchange "diffuses" samples from different spots in the field to nodes that may have never visited these spots. Thus, caches are pro-actively managed so as to capture a local view of the field, which, as best as possible, matches the preference of users for query targets. A query issued locally at a node could then be answered directly by accessing the local cache, or at worst, by accessing the aggregate view in the caches of a small number of direct neighbors.

Paper Outline: The remainder of this paper is organized as follows. In Sections II and III, we provide a statement of the problem, and present our basic assumptions, definitions, and requisite background. In Sections IV and VI, we overview the two alternative solutions to the problem - APR and DPR, respectively. In Section V, using a simplified mobility model, we analyze APR's ability to achieve (say) uniform field coverage using an informed and an uninformed cache management strategy. We confirm APR's premise using extensive simulations, the results of which we present in Section VII. We conclude the paper with a brief discussion of related work in Section VIII and a summary of our findings in Section IX.

\section{Definitions and Problem Statement}

Basic Assumptions: We assume that nodes move independently and autonomously. In particular, node mobility is not driven by the need to effectively sample the field-i.e., the probability of visiting a location in the field is not correlated with the probability for that location to be a query target. We assume that nodes know their locations, either relative or absolute. We assume that storage devoted to our "parasitic" field monitoring service is limited, necessitating the use of a cache management strategy. We also assume that nodes have unique known ID's.

Data Sampling: We assume that mobile nodes sample the field according to a Poisson process. Nodes collect spatiotemporal samples, i.e., every collected sample is associated with an $(x, y)$ coordinates along with a time-stamp to indicate both the sample's location and "age".

Data Freshness: In order to be useful, returned query results should not be "stale". Thus, we assume that a well-defined mechanism exists via which nodes are able to discard obsolete samples (e.g., a time-to-live (TTL) for each sample), or otherwise assign a marginal utility to keeping one sample

\footnotetext{
${ }^{1}$ Even if memory/power are not constrained, the use of flooding would result in extensive network load and increased data dissemination delays due to (or in order to avoid) collisions, with negative implications on timely delivery of data with freshness constraints.
}

versus another - i.e., an aging mechanism. Clearly, choosing the right parameters for aging depends on the stationarity (or time-scale of change) of the target phenomenon sampled by the sensors.

Query Origin and Target: While roaming the field, users may become interested in querying (or reading) the state of a remote location in the field. Such queries are submitted through the query origin (the node associated with the inquirer's device). The remote location that the user is interested in reading is the query target. Different applications may exhibit different distributions of query origins and query targets. While the distribution of query origins may reflect the mobility model of users, ${ }^{2}$ the same cannot be said about the distribution of query targets. In particular, a priori knowledge of the distribution of query targets could be used to improve the performance of the system (e.g., by allowing nodes to give different weights to caching entries based on the spatial coordinates of the entries) [18]. Due to space limitations, in this paper, we address the problem when nodes are equally interested in the whole field (i.e., uniform distribution for query targets). The case when this interest is skewed (e.g., more query targets in the center of the field) is studied in the longer version of this paper [20].

Query Precision: One particularly important parameter of queries is the tolerable inaccuracy in the result. We assume that queries target a specific location in the field along with some desirable precision $(\ell)$, which constrains how far the samples used to answer the query could be from the query target. Introducing query precision allows the support of applications in which queries might target locations in the field where no readings were collected.

Objective and Approaches: Assuming that the distribution of query targets is uniform suggests that the goal of the system would be to achieve uniform field coverage. This goal can be achieved using the DPR and APR approaches motivated in the last section. In the next section, we develop a formal definition of what constitutes "uniform" field coverage. Our approach for that is to use mutual distant sampling of the field, i.e., keep samples that are as far from each other as possible. Section III explains the concept of mutual distant sampling, whereas Section IV explicates how this concept fits in our design. In [20], we show how this technique can be modified to handle non-uniform query distributions.

\section{BACKGRound: Mutually Distant SAmPling}

Teng [30] discusses the NP-hard problem of mutually distant sampling over a metric space and provides an analysis of the performance of a greedy approximation thereof.

Let $\Gamma=(D,\|\|)$ be a metric domain, where \|\| is a nonnegative measure of distance over the domain. We assume that this distance measure satisfies the triangle inequality. Given a positive integer $k$, then $k$-sampling of the domain amounts to finding a set $S$ such that $|S|=k$, and $S$ maximizes the

\footnotetext{
${ }^{2}$ For example, a mobility model that results in higher concentration of users in a particular part of the field will result in a higher number of queries originating from that part of the field.
} 
minimum distance of its points. The minimum distance of $S$ is defined as follows:

$$
\min (S)=\min _{i \neq j}\left\|s_{i}, s_{j}\right\|
$$

i.e., $S$ maximizes the minimum mutual distance between its samples.

Greedy Approximation: Teng [30] proves that the greedy algorithm sketched below provides a 0.5 -approximation to the problem, i.e., $\min _{i \neq j}\left\|s_{i}, s_{j}\right\| \geq 0.5 \times \min _{i \neq j}\left\|t_{i}, t_{j}\right\|$, where $s_{i}, s_{j} \in S, t_{i}, t_{j} \in T$, where $T$ is the optimal solution, and $S$ is the set returned by the greedy algorithm.

Algorithm 1: Input: $\Gamma=(D,\|\|)$, an integer $k \geq 2$.

1) Start with a random point $x \in D$. Let $S=\{x\}$.

2) For $j=2$ to $k$, repeat the following:

2.1) Select the point $y \in D$ such that $y$ maximizes the following function

$$
\psi(S, y)=\min _{q \in S}\|q, y\|
$$

$\psi(S, y)$ defines the distance between a set $S$ and a point $y$ using the measure \|\| , as the minimum distance between $y$ and all points $q \in S$.

2.2) Set $S=S \cup\{y\}$

3) Return $S$.

When $D$ is a set of points $\left\{p_{1}, p_{2}, \ldots, p_{n}\right\} \in \mathbb{R}^{d}$, the complexity of this greedy algorithm is $O\left(k^{2} n\right)$.

\section{Amorphous Placement AND Retrieval (APR)}

APR is a simple scalable algorithm that employs mobility, as opposed to multi-hop communication, whenever possible, to diffuse field samples from field locations to nodes that have never visited these locations. Hence, it improves the local view of each node of the whole field and enables nodes to answer more queries locally saving communication overhead. Avoiding multi-hop communication (as in ad-hoc routing techniques [12], [21]) makes APR efficient in terms of energy consumption and more robust in case of node failures or packet losses, moreover, it saves the overhead of operating an ad-hoc routing protocol. We also show that, interestingly, under limited node mobility, APR results in an informed multihop diffusion of field readings (akin to a selective delaytolerant multi-hop forwarding of these readings).

APR has two main components: (1) sample diffusion, and (2) cache management. Both components work together to enable nodes to optimize the contents of their caches resulting in better matching between the distribution of query targets and locally/nearby cached samples.

Sample Diffusion: The set of samples that are locally cached by a node (i.e., the node's view of the whole field) is a subset of the set of samples this node collects while moving in the field. The latter set is totally defined by the mobility model of nodes, since nodes sample the field along their movement trajectory. However, the workload presented to any node (i.e., targets of queries posed to this node) is independent of the node's trajectory. Hence, we need a mechanism to "decouple" each node's view of the field from its movement trajectory.
This mechanism relies on sample diffusion, whereby upon encountering each other, nodes exchange a small number of samples. This amounts to diversifying the contents of each cache, allowing improved matching of the nodes' local view of the whole field to the query distribution at both nodes.

More specifically, a node $z$ declares its presence to its neighbors by broadcasting a short Hello packet every $\alpha$ seconds. Hello packets contain a compact summary of the cache of $z$ (using a Bloom filter). Upon receipt of such a packet, a neighbor $y$ replies with an Exchange packet: a packet containing $k$ of its samples that failed the Bloom filter test (i.e., node $z$ does not have similar samples and hence its local view of the field would improve by getting these samples). The Exchange packet, likewise, contains a compact summary of $y$ 's cache. Upon receiving an Exchange packet, $z$ adds the received samples to its cache applying the QCCM cache management algorithm described below, if needed. Then it replies to $y$ with a similar packet containing $k$ of its own samples.

Some parameters decisively affect the performance of the sample diffusion process. The first parameter is $\alpha$, the rate of sample diffusion. Slow sample diffusion rates may not specifically help diversifying the contents of caches, resulting in poor performance. While, high diffusion rates may cost too much communication power. The other parameter is the diffusion size $k$. Too small of a value may not be enough to improve performance, while a very large value means more energy consumption. These parameters need to be carefully tuned to optimize the performance of APR.

Query-Cognizant Cache Management: Since the queries originating at a node follow a certain target distribution that is independent of the movement trajectory of this node, it is best to manage the node's cache in a way that makes it store a representation that mirrors this distribution-e.g., when query targets are uniformly distributed, the cache management should strive to cover the entire field as uniformly as possible. To achieve this goal we propose Query-Cognizant Cache Management (QCCM) policy. QCCM is based on maximizing the mutual distance between samples, as explained in Section III. Whenever the cache is full and there are more than one sample to be added to the cache (due to sample diffusion), Algorithm 1 is applied to determine which set of samples should be retained in the cache.

Notice that, in case the query model doesn't follow a uniform distribution over the field, we can always apply a mapping (coordinate transformation) to get the effect of "stretching" (or scaling) the field at the areas of high interest. This would ensure that applying the QCCM algorithm will not evict samples from areas of high interest in favor of other samples covering a less important area. The exact form of this transformation depends on the exact distribution of query targets over the field. We refer interested readers to the extended version of this paper [20] for details of this algorithm when the query distribution is smooth and symmetric in all dimensions (e.g., a bivariate normal distribution), noting that for such a case, one only needs to apply a simple transformation over 
the distances between samples before applying the QCCM algorithm. In the remainder of this paper, unless otherwise noted, we will assume that query targets are uniform over the field.

\section{Effect of Cache Management}

In this section we develop a simple model to gain insight into how much the cache management of multiple mobile nodes affects their collective probability of success in answering queries. We assume that $n$ mobile nodes roam in a twodimensional periodic field (i.e., torus) of size $L \times L$. Each node has a cache of size $c$, where $L^{2} \gg c$. Nodes are given enough time $T$ to sample the entire field ${ }^{3}$. Nodes answer queries uniformly distributed over the field and of precision $\ell$, where we use $L_{1}$ (i.e., Manhattan) distance. The mobile nodes move according to some mobility model, and they sample the field along their movement trajectory, applying a cache management algorithm whenever needed. We model any mobility model through a probability distribution $p_{i j}, \forall(i, j) \in[L, L]$, where $p_{i j}$ is the steady-state probability of any node being in field location $(i, j)$ under that mobility model. A "uniform" mobility model assigns the same probabilities to all field locations, while a "biased" model assigns different probabilities to different field locations (e.g., a random waypoint mobility model results in a higher probability of being in the center of the field). To be amenable to analysis, we assume that any collected sample stays fresh, and so a returned answer is always fresh. This assumption is reasonable if the rate of query/response is much larger than the rate of change in the sampled phenomenon. We relax this assumption in Section VII.

The goal of the model is to compare two cache management algorithms: QCCM, and random cache management (RCM) at steady state-we say that the system reached steady state when all nodes have sampled every location in the field. To focus on the efficiency of the cache management algorithm, we assume that nodes flood the field with their queries, so that cache management decisions done at one node affect the probability of success of queries issued at other nodes. We now introduce two lemmas to help us calculate coverage by each cache management algorithm, which, under the uniform query model assumption, is indicative of the query success ratio.

Coverage of a single sample: Assume a node keeps a sample $e$ at location $(x, y)$, then the coverage of the field attained by keeping $e$ is a function of $\ell$, the query precision. The following Lemma defines coverage of a single sample $R(\ell)$.

Lemma 1: Let $\ell$ denote the query precision. Then, in a twodimensional periodic field, and using the $L_{1}$ distance measure, field coverage attained by keeping any sample (assuming no overlap with coverage from other samples) can be calculated by $R(\ell)=\sum_{i=1}^{\ell} 4 i+1=2 \ell(\ell+1)+1$

Proof: It suffices to notice that on an $L \times L$ torus, the number of neighboring locations at distance exactly $\ell$ from

\footnotetext{
${ }^{3}$ That is $T=O\left(L^{2} \log L\right)$ according to [34]. This is an accurate estimation for $L^{2} \approx 25$.
}

any location equals exactly $4 \ell$, and we add 1 , to account for coverage of the field location where the sample lies.

Optimal Inter-Sampling Spacing (ISS) in 2D torus: We need to answer the question: how can we place $c$ points on a torus of dimensions $L \times L$, such that the minimum mutual distance between any two points is maximized, and what would the optimum distance $S_{\text {opt }}$ in this case. Let's assume for now that $c$ is a square number, i.e., $c=s^{2}$ for some integer $s<L$, and $L$ is a multiple of $s$. Then we can very easily argue that placing the $c$ points uniformly on the field maximizes their mutual minimum distance. In such a case, an optimal algorithm would be one that divides the torus into $s \times s$ squares, then places a point in each square. Selecting the corresponding points in each square yields a minimum ISS of $S_{o p t}=L / s$. The following lemma formalizes this fact.

Lemma 2: In an $L \times L$ torus and given that $c=s^{2}$, if $L>s$ and $L$ is a multiple of $s$, then $S_{\text {opt }} \geq \frac{L}{s}$.

Performance of QCCM: As we discussed above, at steady state, nodes would have sampled the entire field. Recall that QCCM decouples the cache content from the movement trajectory of the nodes. Then we assume that nodes are able to maximize inter-sample spacing, yielding ISS $=S_{o p t}=L / s$. This is always true as long as the mobility model has nonzero probability of visiting all field locations. Since there are $n$ nodes, we know that given any area $A$ of size $=S_{\text {opt }} \times S_{\text {opt }}$, $A$ will host exactly one sample from each node, for a total of $n$ samples in $A$. Coverage of $A$, in this case, corresponds to coverage of the whole field, since the coverage pattern in $A$ is repeated over the rest of the field.

To simplify the analysis, we assume that nodes do not optimize their caches with respect to contents in their neighbors' caches (i.e., nodes do not try to minimize the intersection of coverage achieved by samples in their caches and coverage of samples in their neighbors' caches). Under this assumption, it follows that $A$ has $n$ randomly placed samples. Figure 1 illustrates this setup. Now, consider any field location $\left(l_{i j}\right)$ in $A$, the probability ( $q=\operatorname{Pr}\left[l_{i j}\right.$ covered $]$ ) of covering this location is proportional to the value of $\ell$, and can be calculated as $q=\frac{R(\ell)}{S_{\text {opt }} \times S_{o p t}}$. This follows from the fact that coverage of any field location is related to coverage of one sample. For example, if $\ell=0, l_{i j}$ has only one chance of being covered (i.e., having a sample at $l_{i j}$ ). If $\ell=1$, then $l_{i j}$ has five chances (having a sample at location $l_{i j}$ itself, or having a sample at any of its four neighboring locations), and so on. Now we can view the attempt to cover any field location in $A$ by the $n$ samples, as $n$ independent Bernoulli trials, each with probability of success $q$. Thus, the probability of covering any field location exactly $x$ times (i.e., probability $l_{i j}$ will fall into the coverage area of $x$ different samples) has a binomial distribution and is given by $\operatorname{Pr}[B(n, q)=x]$, where $B(n, q)$ is the Binomial probability. By running a summation of the last quantity for $x=1 \cdots n$, we can obtain the probability of success under QCCM as:

$$
\text { Success }_{Q C C M}=\sum_{x=1}^{n}\left(\begin{array}{l}
n \\
x
\end{array}\right) q^{x}(1-q)^{n-x}=1-(1-q)^{n}
$$




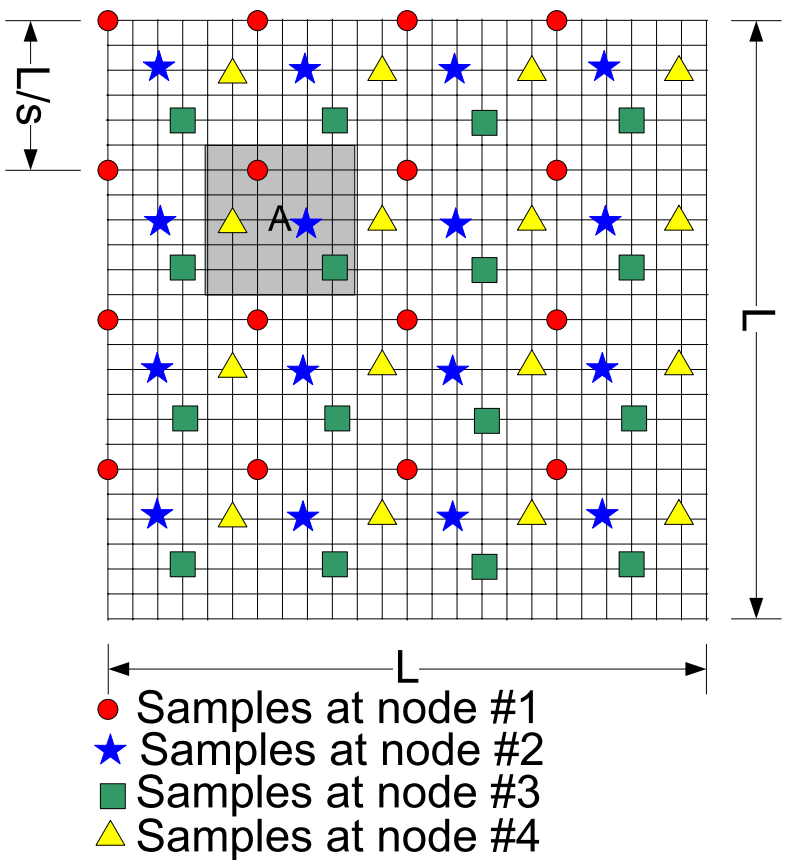

Fig. 1. Idealized field coverage by four nodes applying QCCM. Notice that any area $A=S_{o p t} \times S_{o p t}$ will have exactly one sample from each node.

Performance of RCM: Under RCM, nodes sample the underlying mobility model, hence their cache content will match this distribution. Following the same lines of analysis as we did in QCCM, we have $n$ nodes, each with cache $c$, for a total of $n \times c$ samples in the field. For a given value of $\ell$, let's define $\mathcal{N}_{i j}$ as the set of neighboring locations of $l_{i j}$ within $\ell$ distance units. The probability $\omega_{i j}$ of covering a location $l_{i j}$ can be calculated as: $\omega_{i j}=p_{i j}+\sum_{l_{x y} \in \mathcal{N}_{i j}} p_{x y}$

Hence the probability of $l_{i j}$ being covered exactly $x$ times is given by: $\operatorname{Pr}\left[B\left(n \times c, \omega_{i j}\right)=x\right]$, and the expected number of locations that are covered exactly $x$ times is given by: $\sum_{0 \leq i, j \leq L} \operatorname{Pr}\left[B\left(n c, \omega_{i j}\right)=x\right]$. Then, we can calculate coverage of the field by running a summation for all $x=1 \cdots n \times c$, and the success probability is given by:

$$
\text { Success }_{R C M}=\frac{1}{L^{2}} \sum_{x=1}^{n c}\left(\begin{array}{c}
n c \\
x
\end{array}\right)\left[\sum_{0 \leq i, j \leq L} \omega_{i j}^{x}\left(1-\omega_{i j}\right)^{n c-x}\right]
$$

Figure 2 plots Equations 2 and 3 for two different mobility models depicted in Figure 3 . We have numerically confirmed that both mobility models have no $i, j$ such that $p(i, j)=0$, i.e., nodes can sample the entire field under both models. It is clear that QCCM has a noticeable performance advantage over RCM, as it manages cache content based on the workload, decoupling it from the trajectory of motion of nodes.

\section{Directed Placement and Retrieval (DPR)}

A radically different approach to "amorphous" placement is planned or directed placement. In this approach the system plans the storage location of every group of samples. The storage location is independent of the location of sensors collecting these samples, hence, this approach mandates transporting

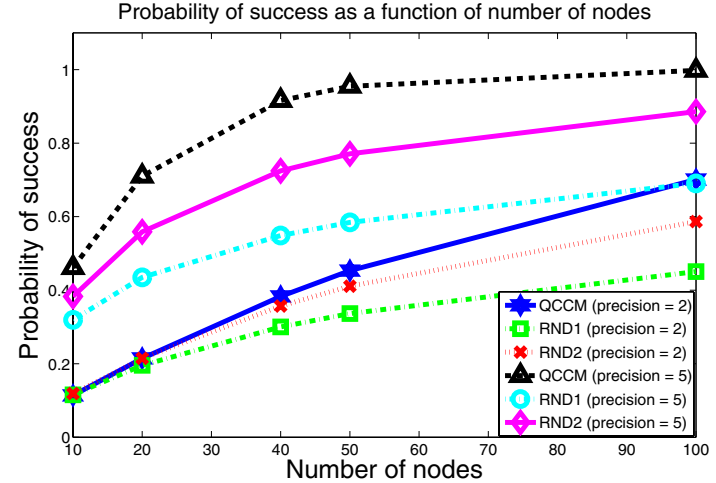

Fig. 2. Effect of cache management algorithm on query success ratio for $n$ mobile nodes. Notice RND2, RCM under mobility model 2 (Figure 3 right), is better than RND1, RCM under mobility model 1 (Figure 3 left), since the earlier is less skewed than the latter.
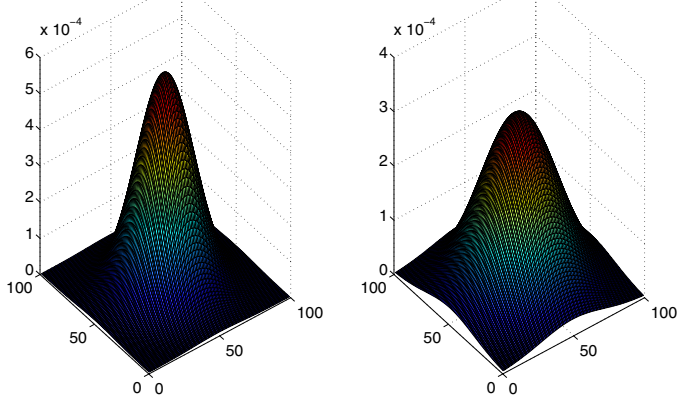

Fig. 3. $p_{i j}$ for the two mobility models used in Figure 2

samples from the collecting sensor(s) to the storage sensor(s). We call the latter the home of the sample. DPR has two main questions to resolve: (1) how to plan sample placement, and (2) how to transport samples from the collecting sensors to the home sensor. Hashing is a widely used technique to answer questions like the first. In systems like [22] and [25], it was proposed to hash samples (based on the sample name) to some location in the field. Nodes closest to that location are considered the home nodes for these samples. To account for mobility, sample replication has been employed to maintain the semantics of the approach (i.e., hashing any sample $e$ to get a field location, sensors closest to a hashed location are the home sensors for $e$ ). Queries are likewise hashed using the same function to get the location where answers to the query should be found. To answer the second question, geographic routing techniques (e.g., GPSR [13]) were employed. Assuming nodes are aware of their own location and that of their neighbors, GPSR can be used to route packets to the node closest to a given location in the field. It is clear that, unlike APR, DPR-like approaches depend on multi-hop communication, which consumes much energy. In this paper, we use a slightly different version from the one described above. Instead of hashing samples and queries to field locations, we hash them to node ID's. It is worth noting that, DPR-like algorithms are not originally designed to handle mobile nodes. However, 
to allow for fair comparison between APR and DPR, we assume that, under DPR, any two nodes $a$ and $b$ route packets (samples, queries, and query answers packets) between them on the optimum route found by applying Dijkstra's algorithm. Dijkstra's algorithm requires instant knowledge of the whole network topology - a piece of information that many realistic systems would lack. Therefore, results reported in this paper should be viewed as providing an upper-bound on any realistic implementation of DPR algorithms. The hashing of samples is based on the sample location. More specifically, we divide the field into Responsibility Regions (RR for short), each region is assigned to a node. All samples collected by any node at the $\mathrm{RR}$ of node $z$ are forwarded to $z$. $z$ manages its cache such that, it keeps samples collected only from its RR. Queries are likewise hashed, based on the query target to get the ID of the home node. Queries are forwarded to their home node, and answers are routed back to inquirer. Having in mind that, nodes only keep samples form their respective RR, the cache management technique used to manage these samples does not have a huge impact on performance. We experimented with both RCM and QCCM, and results were very close. The reason is that the area of RR is usually much smaller than that of the field, that the effect of the cache management is not really noticeable on performance.

\section{ViI. Performance Evaluation}

We evaluated APR and DPR using extensive simulations under a variety of settings. In this section we provide the key results from our experiments, with additional results and extensions available in the longer version of this paper [20].

Simulation Model and Setup: we conducted a set of detailed packet-level simulation experiments, in which we used identical mobility and sampling scenarios for the various approaches. Mobility scenarios for our experiments were generated off line using different mobility models, including the corrected version of the Random Waypoint mobility model [17], the Random Direction model [23] and the Boundless Simulation Area model [7]. Due to space limitations, we only report results for the corrected Random Waypoint model. In our simulations, we set the minimum and maximum speed of motion to $0.1 \mathrm{~m} / \mathrm{sec}$, and $20 \mathrm{~m} / \mathrm{sec}$, respectively.

The sampling process used by mobile nodes follows a Poisson process with exponential inter-arrival time of two seconds; a sample at time $t$ constitutes the sensed value of the field at the current location of the node. We report results of simulating 100 mobile nodes moving in a field of $1400 \mathrm{~m}$ $\times 1400 \mathrm{~m}$, where distance is measured in Euclidean distances. The simulation runs for 5,000 seconds. In the following figures, every point is the average of 20 simulation runs, with 95\% confidence intervals shown. Notice that the confidence intervals are extremely small in most cases. For APR, we set the sample diffusion size $k=4$, and the rate $\alpha=0.005 \mathrm{sec}^{-1}$ (see the longer version of this paper [20] for justification of APR parametrization).

Performance Metrics: The first metric we use is the query success ratio (QSR), which is defined as the ratio between the

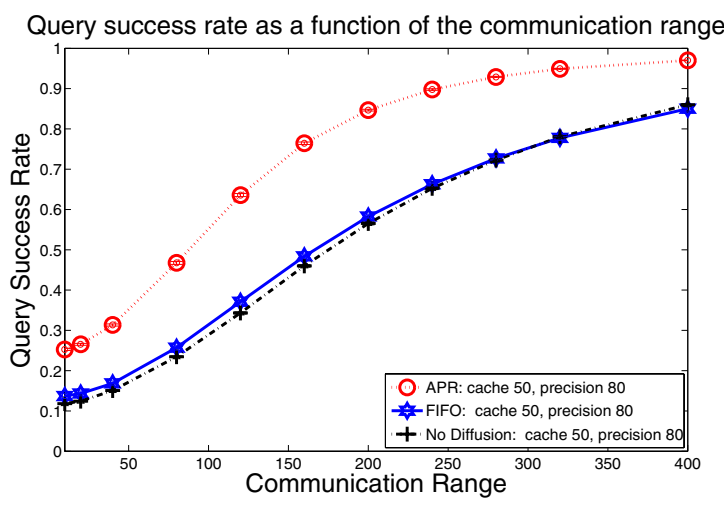

Fig. 4. Effect of APR mechanisms on QSR.

number of successfully answered queries and the number of all queries. To measure efficiency in terms of consumed energy, we compute the number of successfully answered queries per unit energy, to which we refer using Success Per unit Energy (SPE). We use an energy model based on the model presented in [10] (Equations 1 and 2).

Effect of APR Mechanisms: APR has two main mechanisms: sample diffusion, and QCCM. In this subsection, we quantify the effect of each of these mechanisms on APR's performance. To this end, we compare three different versions of APR: (1) APR with both sample diffusion and QCCM, (2) APR with QCCM but no sample diffusion, and 3) APR with sample diffusion, and FIFO cache management. We refer to these using APR, No Diffusion, and FIFO, respectively. Figure 4 shows the QSR of these APR variants as a function of the communication range $r$. These results show that sample diffusion coupled with QCCM achieves the highest QSR. There is a clear difference in performance between APR and FIFO cache management validating our analytical findings in Section V. On the other hand, disabling the sample diffusion mechanisms hinders the performance of APR.

APR versus DPR: In this subsection we compare APR to DPR. To that end, we vary the following parameters: communication range $r$, query precision $\ell$, cache size $c$, TTL, and packet loss probability (PLP). We also study the effect of varying the distribution of queries to a non-uniform distribution, and finally we quantify the effect of mobility (or lack thereof) on both protocols. Due to space limitations, we only show results for communication range, and lack of mobility, and refer the reader to the longer version for the rest of the results [20]. Unless otherwise noted, the default parameters are: $r=160 \mathrm{~m}, \ell=140 \mathrm{~m}, c=50$, TTL $=200$ secs, PLP $=0$, and uniform distribution of queries.

Effect of Communication Range: The communication range $r$ defines the level of connectivity in the network. We argued that DPR would achieve high QSR only when the network is very-well connected, while APR is able to achieve better QSR in less connected networks. Validating our intuition, Figure 5(left) shows query success ratios for APR and DPR using different values for query precision. It is clear that APR 
outperforms DPR for networks with smaller communication range, while the roles are reversed when we increase the communication range. To visualize the impact of network connectivity on QSR for DPR, we also plot the probability of having a connected network as a function of the communication range. This curve is based on the network connectivity model presented in [4]. It is clear that DPR's performance peaks, only when the network is well connected. Increasing the value of $\ell$ (i.e., making precision requirement less strict) helps APR outperform DPR over a wider region of communication ranges. Later, we discuss this effect in more detail. As for SPE, as shown in Figure 5(right), for shorter communication ranges DPR achieves better performance at higher energy consumption level than APR. As the communication range increases, DPR consumes much more energy compared to APR rendering it inefficient in terms of SPE. This is mainly because, unlike APR, DPR depends mainly on multi-hop communication which consumes much energy.

Performance in Static Networks: One might expect that in static networks, APR's performance will deteriorate significantly compared to DPR. In this section, we show that, counter-intuitively, lack of mobility does not impact the general behavior of APR's performance significantly.

In mobile networks, nodes get multiple chances of getting in contact with different neighbors allowing them better sample diffusion and thus an improved view of the entire field. In case of static networks, APR depends, indirectly, on delaytolerant multi-hop dissemination to achieve this effect. To see why this is the case, consider a node $(z)$ at location $\left(x_{z}, y_{z}\right)$ in a completely static network. Due to its immobility, all samples cached by $z$ will be from location $\left(x_{z}, y_{z}\right)$. This will be true until $z$ starts the sample diffusion process with its neighbors. At this point, $z$ will cache samples gathered at locations of its direct neighbors. As the sample diffusion process continues, and QCCM is applied, $z$ will eventually cache samples gathered at neighbors of its direct neighbors, and so on. This effect goes on until $z$ gets a uniform view of the entire field. The combination of QCCM and informed sample diffusion helps to diversify the cache contents of all nodes improving the performance of the entire system. Mobility, only, speeds up this process, especially when the network is not well connected.

The repeated diffusion of samples to nodes farther from the collecting nodes is one form of delay-tolerant multi-hop communication. However, in this case, unlike DPR, nodes on the way get a chance to cache such samples themselves. Figure 6 shows the performance of APR and DPR in a static network as a function of the query precision. The relative trend of APR, seen in mobile settings [20], is still the same (i.e., relaxing the precision constraint improves APR's performance). This accentuates the effectiveness of APR's mechanisms in delivering high performance even in networks with no/limited mobility. Regarding energy efficiency, Figure 6 (right) shows that APR is always more efficient than DPR.

It is worth pointing out that, the effect of network partitioning is more pronounced when there is lack of mobility.
In APR, mobility helps nodes that are temporarily isolated to come in contact with neighbors and exchange valuable samples, which improves the field view at these nodes. When there is no/limited mobility resulting in a partitioned network, disconnected nodes have no such chance and hence their performance deteriorates. This effect is more magnified under DPR, since having persistent network partitions harms the performance of the entire system (due to partitioning the field into RR's and assigning an RR to each node), as opposed to harming the performance of only the group of disconnected nodes, under APR. Another weakness in DPR is that, since some of the RR will not have sensors reside in them, queries about these RR will be always missed. Since APR does not depend on the idea of RR, but rather searches for the sample closest to the query target, the performance of APR for the same queries is decidedly better. The probability of this scenario happening increases as we relax the precision constraint and increase the communication range (see Figure 6).

Summary of Findings: We conclude this section with a summary of findings from all of our experiments.

In this paper, we have shown that: (1) Communication range is the main determinant of DPR's performance: a loosely connected network renders DPR dysfunctional. In contrast, APR features higher resilience to network disconnectivity. (2) APR's performance is not significantly affected by lack of mobility. In fact, when the network is not well connetced, lack of mobility negatively impacts the performance of DPR much more than that of APR. (3) APR is more energy efficient than DPR in almost all situations. In addition to the above results, we also show in the extended version of this paper [20] that: (4) In well-connected networks, queries with tighter precision constraints are better handled by DPR than APR. Relaxing precision constraints improves APR's performance. In loosely-connected networks, APR is better than DPR, even for queries with tight precision constraints. (5) in well-connected networks, when the monitored field values have tight freshness (TTL) constraints, DPR beats APR in handling queries with stringent precision constraints. APR's performance improves as we increase the value of TTL. In loosely-connected networks, the performance of APR dominates that of DPR, independent of freshness (TTL) constraints. (6) Unlike DPR, APR is able to take advantage of increased cache sizes in all settings. (7) APR features much higher resilience to packet losses (and node failures) compared to DPR. (8) Applying a mapping (a linear transformation) to sample locations before feeding them to QCCM, enables APR to deliver superior performance when the query distribution is non-uniform over the field.

\section{RELATED WORK}

There have been extensive research on data management and query resolution in sensor networks. Applications where sensors are mobile and produce large-size samples (e.g., cameras) make these problems more challenging. Due to space limitations, we restrict our attention to only a representative 

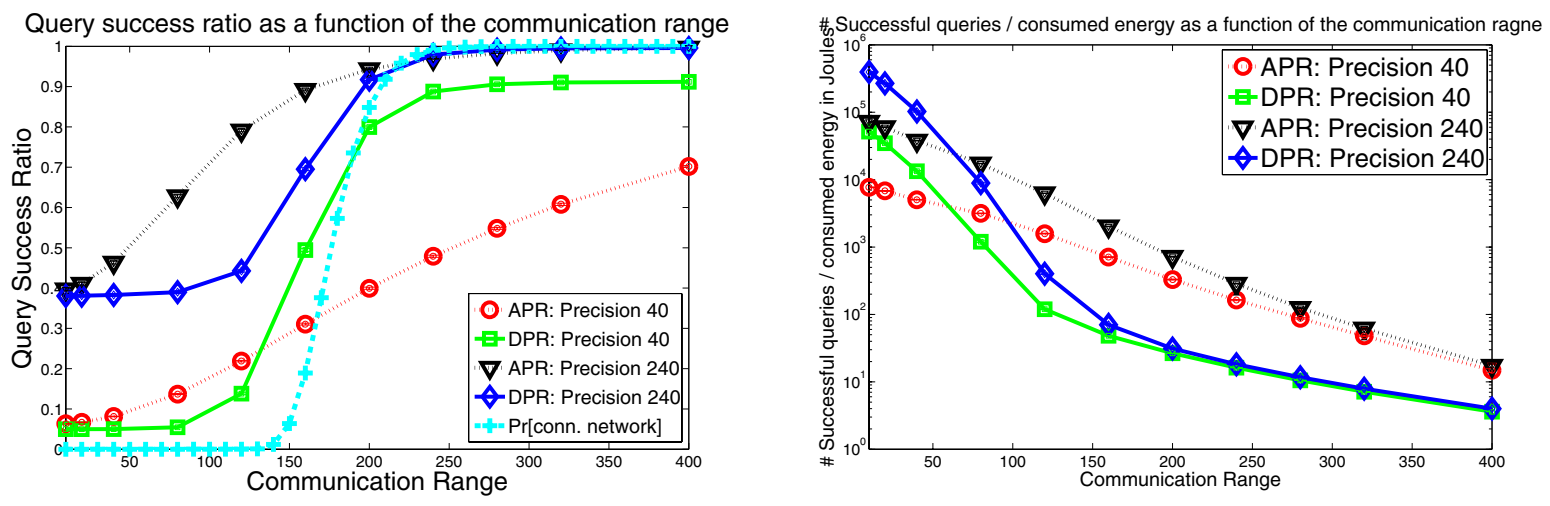

Fig. 5. Effect of communication range: Query success ratio (left) and Query success ratio per unit of energy (right).
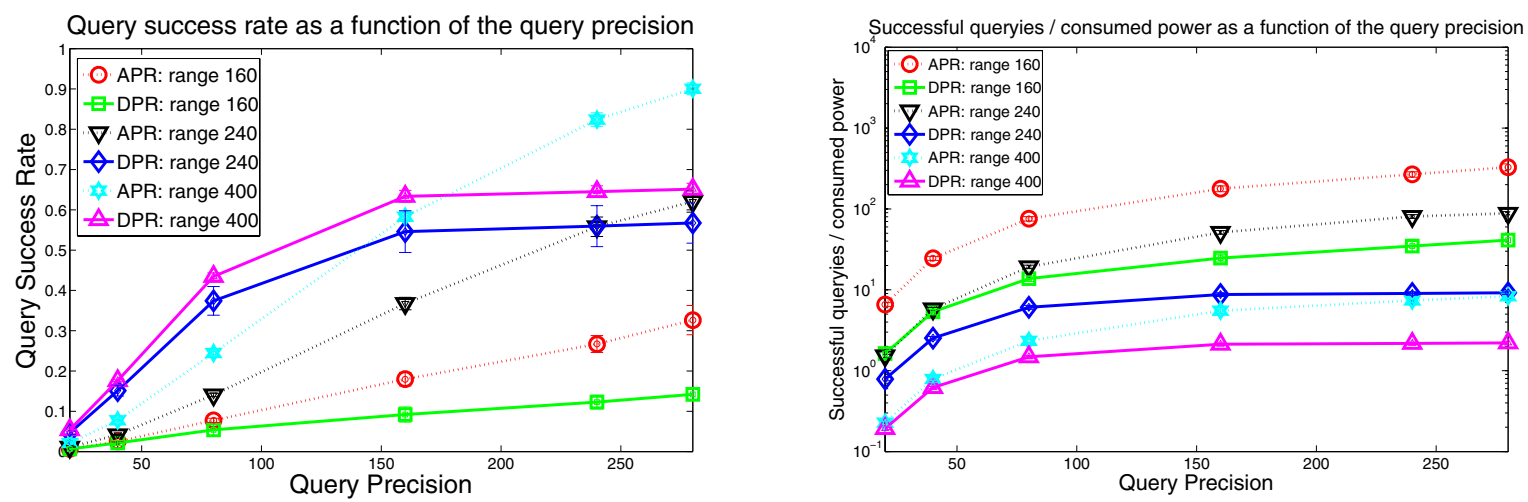

Fig. 6. Effect of query precision in a static network: Query success ratio (left) and Query success ratio per unit of energy (right).

sample of related research, which we broadly categorize based on whether the network or the users (sinks) are mobile.

Static/mobile network, static sinks: Data Centric Routing (DCR) and Data Centric Storage (DCS) fall into this category. DCR, such as Directed Diffusion [11], employs flooding. The overhead of flooding is amortized assuming long-running queries. A sink floods its query/interest, and targeted sensors respond. In our APR scheme, each node is able to answer queries locally, or at worst, using limited-scope flooding, since each node actively builds a view of the entire field that matches the spatial distribution of the query targets.

DCS [27] attempts to avoid flooding altogether, hence is suitable for one-shot queries. DCS employs hashing to associate a data item with a specific location in the field. A geographic routing protocol, such as GPSR [13], is used to transport a query/answer for a data item. We compared APR with DPR, which is basically a DCS approach.

Mobility challenges the design of both DCR and DCSit continually changes the topology underneath the routing protocol. Other proposals, such as data mules [26], smart tags [3] and mobile relays [32], employ mobile elements as relays among static nodes. These schemes target delay-tolerant applications, which is not the focus of this paper. Another delay-tolerant scheme was proposed by Small et al. [28] for a whale monitoring application.

Static network, mobile sinks: Both TTDD [35] and SEAD
[15] fall into this category. They target long-running queries from mobile users. Essentially, these schemes can be thought of as a hierarchical extension to Directed Diffusion, whereby the effect of sink mobility is localized.

Mobile network, mobile sinks: The work by Zaho et al. [37] and Lee et al. [16] fall into this category. The first employs powerful message ferries to act as relays. In the latter proposal, each node keeps track of its recent contacts, along with their sensed events, and employs last-encounter routing to locate a target node. In a similar setting of delay-tolerant applications, Wang et al. [33] employs history-based forwarding and buffer management.

Our proposed APR protocol also fall into this category, albeit its suitability for delay-sensitive applications. APR does not require external mobile elements, such as ferries or data mules. And APR takes a different, proactive approach to improve query performance. The key idea in APR is that it decouples the negative effects of uncontrolled mobility on query performance, by making cache management cognizant of the query profile. Thus, queries can be readily answered from the field view (samples) stored in the local cache or very few neighboring caches.

Another related body of work, is data management in ad hoc networks [36], [8]. The main difference between these efforts and ours is that: usually, in ad hoc networks, the set of data objects is limited with a known source for every object, which 
is not the case in mobile sensor networks, since any node can sample any field location. Moreover, correlation between different data objects are usually ignored. Hara et al. consider this correlation in [9]. However, the correlation structure they consider is random. In our case, the correlation between samples is manifested in utilizing samples to answer queries targeting close-by field locations. Hence, the correlation is not random and has a physical interpretation.

The sample diffusion idea used by APR resembles gossiping and randomized rumor routing [14], [5], [31]. In these efforts multi-hop routing of delay-tolerant data is avoided and mobility is deployed instead. However, as we have shown, APR is flexible enough to resort to delay-tolerant multi-hop forwarding when the need arises. The sample diffusion process also borrows ideas from the summary cache [6] by Fan et al. to maximize its gain.

\section{CONCLUSION}

In this paper, we presented a proactive approach, APR, that amorphously places and diffuses sensor data collected by autonomously mobile nodes, allowing nodes (and node neighborhoods) to compile an integrated view of the monitored field of interest, in anticipation of freshness-constrained and precision-constrained queries thereof. A salient feature of APR is that it enables the management of the nodes' cache content in such a way so as to match the distribution of query targets, regardless of the distribution of the locations that are collectively visited (and sensed). Given a uniform distribution of queries over the space, we demonstrated, by analysis and extensive simulations, how query performance improves under an informed (query-aware) diffusion of sensory samples that maximizes the minimum distance between samples in a node's cache. Our current work is focused on the development of a general transformation that allows APR to handle arbitrary distributions of query targets - and not only the symmetric distributions we considered in this work.

\section{REFERENCES}

[1] Lifeguard, wearable wireless physiological monitor. http://lifeguard.stanford.edu/.

[2] M. Ali and Z. A. Uzmi. CSN: A network protocol for serving dynamic queries in large-scale wireless sensor networks. In CNSR'04, 2004.

[3] A. Beaufour, M. Leopold, and P. Bonnet. Smart-tag based data dissemination. In WSNA '02.

[4] C. Bettstetter. On the connectivity of wireless multihop networks with homogeneous and inhomogeneous range assignment. In IEEE Vehicular Technology Conf. (VTC), Vancouver, BC, Canada, September 2002.

[5] R. R. Choudhury. Brownian gossip: Exploiting node mobility for diffusing information in wireless networks. In StoDis'05.

[6] L. Fan, P. Cao, J. Almeida, and A. Z. Broder. Summary cache: a scalable wide-area web cache sharing protocol. IEEE/ACM Trans. Netw. 8(3):281-293, 2000.

[7] Z. Haas. A new routing protocol for the reconfigurable wireless networks. In ICUPC'97.

[8] T. Hara. Effective replica allocation in ad hoc networks for improving data accessibility. In Infocom '01, pages 1568-1576, 2001.

[9] T. Hara, N. Murakami, and S. Nishio. Replica allocation for correlated data items in ad hoc sensor networks. SIGMOD Rec., 33(1):38-43, 2004.

[10] W. R. Heinzelman, A. Chandrakasan, and H. Balakrishnan. Energyefficient communication protocol for wireless microsensor networks. In HICSS 'OO.
[11] C. Intanagonwiwat, R. Govindan, and D. Estrin. Directed diffusion: a scalable and robust communication paradigm for sensor networks. In MobiCom '00, pages 56-67, 2000.

[12] D. B. Johnson, D. A. Maltz, and J. Broch. Dsr: the dynamic source routing protocol for multihop wireless ad hoc networks. pages 139$172,2001$.

[13] B. Karp and H. T. Kung. Gpsr: greedy perimeter stateless routing for wireless networks. In MobiCom ' 00 .

[14] A.-M. Kermarrec, L. Massouli, and A. J. Ganesh. Probabilistic reliable dissemination in large-scale systems. IEEE Trans. Parallel Distrib. Syst. 14(3):248-258, 2003.

[15] H. S. Kim, T. F. Abdelzaher, and W. H. Kwon. Minimum-energy asynchronous dissemination to mobile sinks in wireless sensor networks. In SenSys '03, pages 193-204, New York, NY, USA, 2003. ACM Press.

[16] U. Lee, E. Magistretti, B. Zhou, M. Gerla, P. Bellavista, and A. Corradi. Efficient data harvesting in mobile sensor platforms. In PERCOMW '06.

[17] G. Lin, G. Noubir, and R. Rajamaran. Mobility models for ad-hoc network simulation. In INFOCOM, 2004.

[18] C. Lu, G. Xing, O. Chipara, C.-L. Fok, and S. Bhattacharya. A spatiotemporal query service for mobile users in sensor networks. In ICDCS 05.

[19] P. Lukowicz, U. Anliker, J. Ward, G. Trster, E. Hirt, , and C. Neufelt. Amon: A wearable medical computer for high risk patients. In $I S W C^{\prime} 02$.

[20] H. Morcos, A. Bestavros, and I. Matta. Amorphous placement and informed diffusion for efficient field monitoring by autonomusly mobile sensors. Technical Report BUCS-TR-2007-008, Computer Science Department, Boston University, 111 Cummington Street, Boston, MA 02135, June 2007.

[21] C. Perkins, E. Belding-Royer, and S. Das. Ad hoc on-demand distance vector (aodv) routing. 2003.

[22] S. Ratnasamy, B. Karp, L. Yin, F. Yu, D. Estrin, R. Govindan, and S. Shenker. Ght: a geographic hash table for data-centric storage. In WSNA '02, pages 78-87, New York, NY, USA, 2002. ACM Press.

[23] E. M. Royer, P. M. Melliar-Smith, and L. E. Moser. An analysis of the optimum node density for ad hoc mobile networks. In ICC'01.

[24] R. S., F. P., H. M., K. R., and S. S. A scalable content-addressable network. In SIGCOMM '01.

[25] K. Seada and A. Helmy. Refereed poster: Rendezvous regions: A scalable architecture for service provisioning in large-scale mobile ad hoc networks. In ACM SIGCOMM, 2003.

[26] R. Shah, S. Roy, S. Jain, and W. Brunette. Data mules: Modeling a three-tier architecture for sparse sensor networks. In IEEE SNPA'03.

[27] S. Shenker, S. Ratnasamy, B. Karp, R. Govindan, and D. Estrin. Datacentric storage in sensornets. SIGCOMM Comput. Commun. Rev., 33(1):137-142, 2003

[28] T. Small and Z. J. Haas. The shared wireless infostation model: a new ad hoc networking paradigm (or where there is a whale, there is a way). In MobiHoc '03.

[29] I. Stoica, R. Morris, D. Karger, M. F. Kaashoek, and H. Balakrishnan. Chord: A scalable peer-to-peer lookup service for internet applications. In SIGCOMM '01.

[30] S.-H. Teng. Low energy and mutually distant sampling. J. Algorithms, 30(1):52-67, Jan 1999.

[31] S. Voulgaris, M. Jelasity, and M. van Steen. A robust and scalable peerto-peer gossiping protocol. In 2nd Int'l Workshop Agents and Peer-toPeer Computing, LNCS 2872,, Springer, 2003.

[32] W. Wang, V. Srinivasan, and K.-C. Chua. Using mobile relays to prolong the lifetime of wireless sensor networks. In MobiCom '05.

[33] Y. Wang and H. Wu. Dft-msn: The delay/fault-tolerantmobile sensor network for pervasive information gathering. In INFOCOM '06.

[34] G. H. Weiss. Aspects and Applications of the Random Walk. Elsevier Science B.V., North-Holland, 1994.

[35] F. Ye, H. Luo, J. Cheng, S. Lu, and L. Zhang. A two-tier data dissemination model for large-scale wireless sensor networks. In MobiCom '02, pages 148-159, New York, NY, USA, 2002. ACM Press.

[36] L. Yin and G. Cao. Supporting cooperative caching in ad hoc networks. In INFOCOM'04.

[37] W. Zhao, M. Ammar, and E. Zegura. A message ferrying approach for data delivery in sparse mobile ad hoc networks. In MobiHoc ' 04. 